\title{
EVALUACIÓN DEL COMPORTAMIENTO GERMINATIVO DE TRES ESPECIES VEGETALES NATIVAS CON POTENCIAL ORNAMENTAL DEL CENTRO - NORTE SANTAFESINO
}

\author{
Ghío, N. ${ }^{1} ;$ Marinoni, L. ${ }^{2}$; CAStro, D. ${ }^{3}$ Zabala, M. ${ }^{2}$; \\ Micheloud, N. G. ${ }^{3} \&$ BUYATTI, M. ${ }^{3}$
}

\begin{abstract}
RESUMEN
La flora nativa posee un enorme potencial paisajístico que ha sido poco explorado. En el presente trabajo se han estudiado aspectos relacionados con las semillas y comportamiento germinativo de tres especies vegetales nativas del centro - norte santafesino con potencial ornamental priorizadas para su introducción a la producción en viveros en el marco de las acciones del Programa de Documentación, Conservación y Valoración de la Flora Nativa (PRODOCOVA). Las especies en estudio corresponden a "Sangre drago" Croton urucurana Baill., "Horquetero" Tabernaemontana catharinensis A. DC. y “Ojo de muñeca” Paullinia elegans Cambess. Para C. urucurana se halló una temperatura óptima a temperaturas alternantes de $20-30{ }^{\circ} \mathrm{C}$, mientras que para P. elegans y $T$. catharinensis, temperatura constante a $25{ }^{\circ} \mathrm{C}$ determinó el mayor porcentaje de germinación. Se identificaron para todas las especies el momento para el recuento inicial y final del porcentaje de germinación. Adicionalmente se observó que semillas de P. elegans son recalcitrantes. Estos resultados son de gran utilidad para la introducción a cultivo de las especies en estudio.
\end{abstract}

Palabras clave: paisajismo, semillas, viverización, porcentaje de germinación.

\footnotetext{
1.- Cientibecario Proyecto CAI+D Orientado 2016 CS No 632/17. III. 3.1.

2.- Cátedra Genética y Mejoramiento Vegetal y Animal. Facultad de Ciencias Agrarias. Universidad Nacional del Litoral. Kreder 2805. (3080) Esperanza, Santa Fe, Argentina.

3.- Cátedra Cultivos Intensivos. FCA (UNL). Email: mbuyatti@fca.unl.edu.ar

Manuscrito recibido el 14 de abril de 2020 y aceptado para su publicación el 23 de diciembre de 2020.
}

Ghio, N.; Marinoni, L.; Castro, D.; Zabala, M.; Micheloud, N. G. ; Buyatti, M. Evaluación del comportamiento germinativo de tres especies nativas con potencial ornamental del centro - norte santafesino.

FAVE - Ciencias Agrarias 20 (1): 175-188. CC BY-NC-SA 4.0 


\begin{abstract}
Evaluation of the germinative behaviour of three native species with ornamental potencial in the centre-north of Santa Fe.

The native flora has an enormous landscaping potential not fully explored yet. In the present work, aspects related to seeds and germination behavior of three native plant species from central-northern Santa Fe with ornamental potential, prioritized for their introduction to nursery production in the framework of the actions of the Program for Documentation, Conservation and Valuation of Native Flora (PRODOCOVA), have been studied. The studied species were "Sangre drago" Croton urucurana Baill, "Horquetero” Tabernaemontana catharinensis A. DC., and “Ojo de muñeca” Paullinia elegans Cambess. For C. urucurana alternating temperatures of $20-30{ }^{\circ} \mathrm{C}$ were found as optimal temperature, while for P. elegans and $T$. catharinensis, constant temperature at $25{ }^{\circ} \mathrm{C}$ determined the highest percentage of germination. Additionally, the initial and final count of the germination percentage were determined for each species. Finally, it was also noted that seeds of $P$. elegans are recalcitrant. These results provide the basis for the introduction to cultivation of the studied species.
\end{abstract}

Key words: Landscaping, seeds, nursery, germination rate.

\section{INTRODUCCIÓN}

Las plantas son parte fundamental del sostén de los ecosistemas verdes de la Tierra, pero también son esenciales para la supervivencia humana. Es conocido que las plantas proporcionan la mayor parte de los alimentos que consumimos (directa o indirectamente), nuestras medicinas, ropa, edificios y hasta el aire que respiramos; también embellecen nuestra vida cotidiana. El aprovechamiento ornamental de la flora nativa se limita a pocas especies, si se compara con la cantidad y diversidad de especies silvestres que podrían incorporarse al cultivo. Características como forma, color del follaje o una floración vistosa, hacen de las plantas nativas un elemento potencialmente valioso para jardinería y paisajismo, lo que justifica el estudio de sus probabilidades de propagación y difusión. Actualmente, con el creciente impacto hu- mano sobre las plantas, animales y hábitats naturales, nuestras acciones pueden hacer una gran diferencia si las plantas son parte de la civilización ecológica para el futuro. Es de suma importancia el poder estudiarlas y utilizarlas responsablemente, tanto como científicos, así como miembros de las sociedades humanas (Carreras et al., 2003; Rendón Correa y Fernández Nava, 2007; Knapp, 2017).

La producción mundial de plantas ornamentales y sus industrias subsidiarias, generan anualmente un valor agregado aproximado de entre 250 a 400 mil millones de dólares, alrededor del 0,5\% del producto bruto mundial. Para Argentina se estima que el valor bruto de la producción está entre 220 a 240 millones de dólares (Botto y Mata, 2014). En cuanto a la diversidad biológica, los recursos genéticos nativos y los mercados globales asociados a ello, forman la base de una proporción significativa de la activi- 
dad económica mundial. Una estimación de las ventas de los mercados globales anuales para el área de los productos hortícolas ornamentales derivados completamente de recursos genéticos se encuentra entre 16 y 19 billones de dólares (Morisigue et al., 2012).

La viverización de especies nativas con fines paisajísticos es una temática que ha adquirido relevancia en los últimos años. En este sentido, se ha conformado en 2016 la Red de Viveros de Plantas Nativas (REVINA) que reúne diferentes actores involucrados en la identificación, valoración y producción de plantas nativas. Adicionalmente, en el marco de las acciones del Programa de Documentación, Conservación y Valoración de la Flora Nativa (PRODOCOVA), que se desarrolla en la FCA-UNL desde el año 2012, se han identificado y colectado especies nativas con diferentes potencialidades agronómicas en las que se incluyen especies con valor ornamental. Estas acciones contribuyen a la conservación y a la valoración de la flora autóctona.

La propuesta que se presenta, se enmarca en un contexto internacional en el que el paisajismo tiende a integrar las áreas naturales a las urbanas, resaltando el valor de las especies silvestres con la finalidad de recuperar áreas degradadas, aprovechar los servicios ambientales que ofrecen (p.e. polinización), mantener la identidad del paisaje natural y conservar germoplasma autóctono.

Para este trabajo se seleccionaron para su estudio tres especies nativas, "Sangre drago” Croton urucurana Baill. (Euforbiaceae); "Horquetero" Tabernaemontana catharinensis A. DC. (Apocynaceae) y "Ojo de muñeca” Paullinia elegans Cambess. (Sapindaceae), dado sus múltiples usos potenciales, incluido el ornamental (para más detalles del valor ornamental de las espe- cies en estudio se puede consultar el Banco de Imágenes Irupé de la FCA; https://www. fca.unl.edu.ar/prodocova/IRUPE).

Sangre drago (Croton urucurana Baill.) es una especie de las regiones tropicales y subtropicales de Sudamérica, frecuente en Brasil, Paraguay, Bolivia, Uruguay y Argentina; y en esta última, en las provincias de Misiones, Corrientes, Entre Ríos, Formosa, Chaco y Santa Fe. Habita en suelos arenosos y húmedos de los bosques de la costa santafesina, próximo a los cursos de agua e interior de islas (Pensiero y De la Peña, 1999; Flora Argentina, 2019). Su potencialidad ornamental yace en sus vistosas hojas que, durante el otoño, se tornan de color naranja a rojizas. Debido a que es un árbol pequeño, vistoso y de follaje caduco, es muy recomendable para arbolado urbano (Pensiero y De la Peña, 1999). Se la considera una planta pionera y rústica, adaptada a terrenos muy húmedos y pantanosos, es óptima para plantíos en áreas ribereñas degradadas (Lorenzi, 1992). Se menciona a $C$. urucurana, como una especie con semillas ortodoxas (i.e. sobreviven a períodos prolongados de desecación y bajas temperaturas durante su conservación ex situ) (Mori et al., 2012), mientras que en germinación existe evidencia de diferencias en la capacidad germinativa de semillas de diferentes colores y ante diferentes tratamientos pre-germinativos en estudios llevados a cabo en poblaciones nativas del sur de Brasil (Durigan et al., 1997; Pinho et al., 2009).

Horquetero o Palo Víbora (Tabernaemontana catharinensis A. DC.), es un árbol pequeño de follaje verde brilloso, con flores blancas perfumadas contortas que recuerdan a los jazmines. Crece desde Brasil hasta el noreste de Argentina, en las provincias de Jujuy, Formosa, Chaco, Santa Fe, Misiones, Corrientes, Entre Ríos, y Buenos 
Aires, y además en Uruguay. Es frecuente en los bordes y claros de las selvas siendo su límite austral el norte de Santa Fe y Entre Ríos (Biloni, 1990). Pionera, frecuente en los matorrales y bosques secundarios del Chaco Oriental, se establece rápidamente después de aprovechamientos forestales que alteran el bosque alto, con alto poder de brotación a partir de raíces. Florece desde noviembre a enero y fructifica de febrero a abril (Gimenez y Moglia 2003). Su potencialidad como ornamental radica en que es un árbol de porte medio, de buen follaje, siempre verde, floración vistosa y fácil propagación. Estudios realizados por Afonso (2016), determinaron temperaturas óptimas de germinación a 25 y $30^{\circ} \mathrm{C}$ en poblaciones de T. catharinensis nativas del rio Grande do Sul, independientemente del régimen de luz. Adicionalmente, demostraron que las semillas poseen un comportamiento ortodoxo.

Ojo de muñeca (Paullinia elegans Cambess.), es una planta de hábito tipo liana, ampliamente distribuida desde Colombia, Bolivia, Paraguay, Uruguay hasta el NE de Argentina, donde crece en las provincias de Chaco, Formosa, Misiones, noroeste de Santa Fe, Corrientes y Entre Ríos. Vegeta en el borde de la selva marginal, pero también aparece en la vegetación secundaria. Florece en octubre-junio, con frutos en diciembre-agosto, aunque son más abundantes en el verano (Pensiero y De la Peña, 1999; Verde Chaco, 2011). Lo vistoso de esta especie son los frutos rojizos que, a la madurez, se abren dejando a la vista las semillas negruzcas recubiertas de un arilo blanquecino, que da el aspecto a un ojo de muñeca, tal como se la conoce vulgarmente a la especie.

Resumiendo, la evidencia relacionada al comportamiento germinativo de las especies establece que, en $C$. urucurana son necesarios tratamientos pre-germinativos para promover la germinación de las semillas, en particular, semillas color gris las que, además, son ortodoxas. En T. catharinensis hay evidencia de que sus semillas son ortodoxas y que germinan sin necesidad de tratamientos pre-germinativos. En P. elegans, se desconoce el comportamiento de sus semillas al almacenamiento a baja humedad y temperatura, aunque experimentos preliminares indican que se tratarían de semillas de comportamiento recalcitrante, y se conoce que no son necesarios tratamientos pre-germinativos. Aunque se han reportado temperaturas óptimas de germinación para las especies en estudio, esa información no pertenece a poblaciones nativas de la región santafesina, incluso de Argentina.

Por lo antes mencionado, en el presente trabajo se evaluó la temperatura óptima de germinación de poblaciones nativas del centro - norte santafesino de Croton urucurana, Tabernaemontana catharinensis y Paullinia elegans. Adicionalmente, se ensayaron diferentes tratamientos pre-germinativos en $C$. urucurana y se evaluó el comportamiento al almacenamiento a baja humedad en semillas de $P$. elegans, información de suma importancia para la introducción de dichas especies al mercado ornamental.

\section{MATERIALES Y MÉTODOS}

\section{Material vegetal}

Las colectas fueron realizadas por los responsables del Banco de Germoplasma “Ing. Agr. José M. Alonso” y docentes de la Cátedra de Cultivos Intensivos de la Facultad de Ciencias Agrarias de la Universidad Nacional del Litoral, en diferentes áreas de su distribución natural en los departamentos La Capital, Juan de Garay y San Javier, de 
la provincia de Santa Fe, en la zona denominada Albardón Costero, perteneciente a la región fitogeográfica del Delta e islas del Paraná (Biasatti et al, 2016) (Figura 1). Se colectaron frutos de tres poblaciones de $C$. urucurana, en el Dpto. La Capital, distrito Arroyo Leyes, a lo largo de un corredor de $4 \mathrm{~km}$; dos poblaciones de $T$. catharinensis, en el Dpto. Garay, en los alrededores de la localidad de Cayastá; y cinco poblaciones de $P$. elegans en el Dpto San Javier, a lo largo de la ruta provincial $\mathrm{N}^{\circ} 1$ y la costa del río San Javier, entre el paraje Los Jacintos y la localidad de Alejandra. Todas las plantas de las poblaciones colectadas fueron geo- rreferenciadas, incluyendo material para el Herbario Arturo E. Ragonese (“SF”). El trabajo de selección y georreferenciación fue realizado, en primera instancia, en el marco de misiones de colecta del PRODOCOVA y luego durante diversos viajes de prospección durante los años 2017 y 2018 realizados por la Cátedra de Cultivos Intensivos, en el marco del Proyecto CAI+D Orientado 2016. Los viajes de colectas fueron realizados en temporada otoñal (marzo - abril), ya que es la época ideal para la cosecha de semillas de estas especies. Cabe aclarar que los meses de marzo y abril para ambos años de colecta, coincidieron con importantes

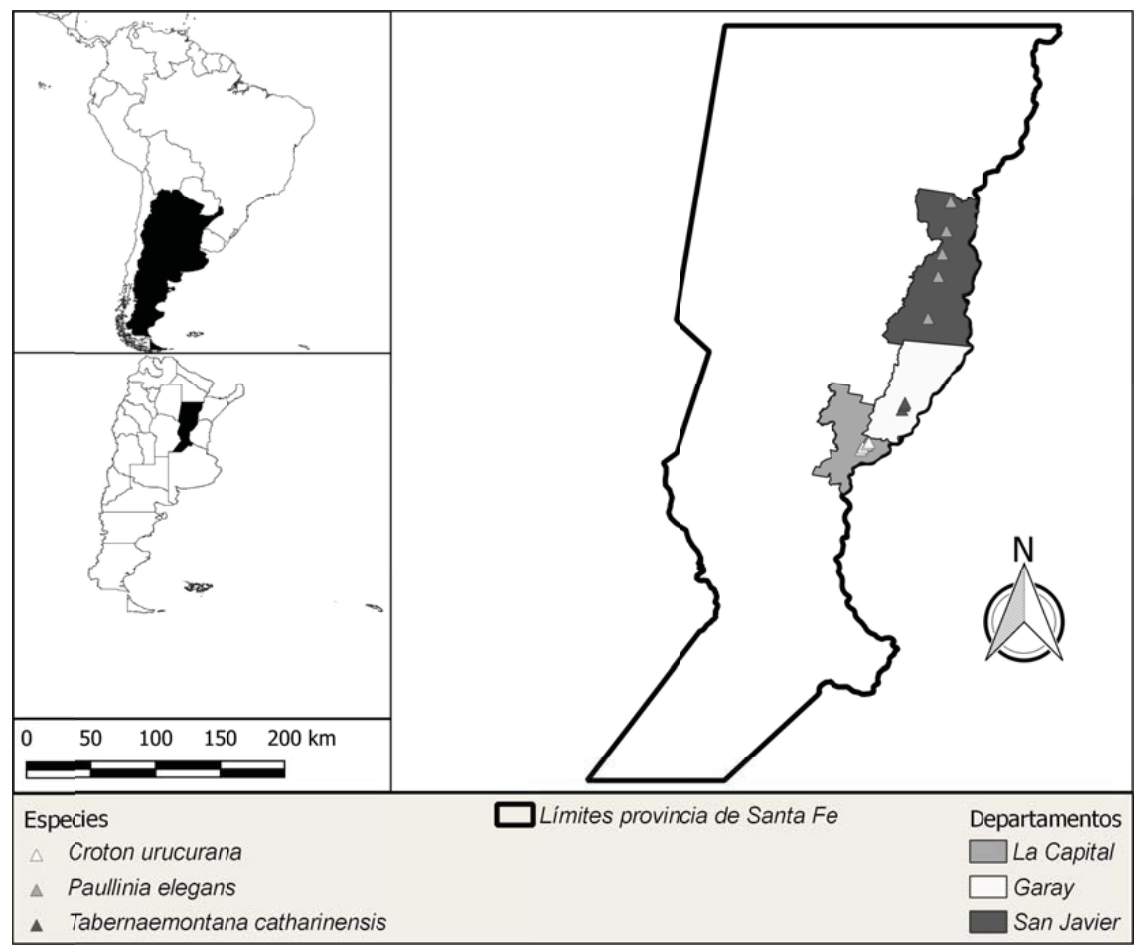

Figura 1: Mapa de la provincia de Santa Fe, donde se observa la ubicación geográfica de las colectas realizadas para cada una de las especies en estudio

Figure 1: Map of the province of Santa Fe, showing the geographical location of the collections made for each of the species under study 
precipitaciones y altas temperaturas. Esto incidió en la calidad de la semilla colectada, especialmente para el caso de P. elegans $\mathrm{y}$ de $T$. catharinensis que, al tener frutos carnosos, estos estaban muy atacados por larvas de insectos.

Los frutos de cada una de las especies en estudio fueron procesados luego de la colecta para separar las semillas. En el caso de C. urucurana, este posee un fruto seco indehiscente denominado esquizocarpo. Estos fueron trillados por fricción para liberar las semillas; adicionalmente, se seleccionaron semillas de color gris las que, según Scalon et al. (2012), lo cual fue comprobado con ensayos preliminares en nuestro grupo de trabajo, son las únicas con capacidad de germinar. Los frutos de $P$. elegans (cápsulas carnosas) se abren a la madurez, dejando libres las semillas, las que poseen un arilo carnoso blanquecino que fue removido con papel absorbente ya que posee un alto contenido de carbohidratos y favorece la proliferación hongos. Por otro lado, T. catharinensis posee a la madurez un fruto seco pluriseminado (folículo), dehiscente, cuyas semillas poseen un arilo carnoso y jugoso que fue retirado con papel absorbente y puesto a secar en ambiente de baja humedad para evitar la proliferación de hongos. En todos los casos se eliminaron semillas vanas, malformadas, rotas o atacadas por hongos. Para cada una de las especies, se trabajó con muestras masales de semillas (mezcla en iguales proporciones de semillas de las poblaciones colectadas), se determinó el peso de 100 semillas (g) y se evaluó el comportamiento germinativo (Hartmann et al., 2002).

\section{Ensayos de germinación}

Croton urucurana: Se ensayaron diferentes tratamientos pre-germinativos combinados con diferentes temperaturas de germinación. Los tratamientos pre-germinativos consistieron en: 1) inmersión en agua durante 2 horas; 2) inmersión en agua durante 12 horas; 3) inmersión en solución con ácido giberélico [(GA) 200 mg.L-1] durante 2 horas; 4) inmersión en solución con GA (200 mg.L-1) durante 12 horas; 5) control (sin tratamiento pre-germinativo). Luego de realizados los diferentes tratamientos pre-germinativos, se incubaron las semillas a diferentes temperaturas: 1) constante a $25{ }^{\circ} \mathrm{C}, 2$ ) constante a $30^{\circ} \mathrm{C}$ y 3) alternancia de temperaturas 20 $30{ }^{\circ} \mathrm{C}$ (8/16 horas). De esta manera quedaron definidos 15 tratamientos derivados de la combinación de los cinco tratamientos pre-germinativos y las tres temperaturas de germinación. Para cada tratamiento, se utilizaron cuatro repeticiones, de 25 semillas dispuestas en cajas de Petri (unidad experimental). Se efectuaron recuentos periódicos durante 30 días, para establecer la fecha óptima que defina el porcentaje de germinación inicial y final. Adicionalmente, se tomó una muestra de 50 semillas a la que se le efectuó la prueba de viabilidad a través del test de Tetrazolio (Moore, 1985).

Paullinia elegans: se evaluó el comportamiento germinativo a diferentes temperaturas contantes: 1) $20{ }^{\circ} \mathrm{C}$, 2) $25^{\circ} \mathrm{C} \mathrm{y} \mathrm{3)}$ $30{ }^{\circ} \mathrm{C}$, que definieron los tratamientos. Se efectuaron recuentos periódicos durante 30 días, para establecer la fecha óptima que defina el porcentaje de germinación inicial y final. Para esta evaluación se utilizaron semillas procesadas inmediatamente después de la colecta, ya que estudios preliminares (datos no publicados) determinaron un posible comportamiento recalcitrante de sus semillas.

Revista FAVE - Ciencias Agrarias 20 (1) 2021 
El principal determinante del comportamiento recalcitrante de las semillas es la pérdida de viabilidad ante la desecación o disminución del contenido hídrico de las semillas, por debajo de $20 \%$ (Willian 1991). Para determinar el comportamiento al almacenamiento en P. elegans, se tomó una muestra de 50 semillas procesadas inmediatamente después de la recolección y se determinó el porcentaje de humedad. Para ello, se registró el peso inicial de la muestra (PI) (g), con balanza de precisión (0.0001 g), se llevó luego a estufa a $100{ }^{\circ} \mathrm{C}$ hasta peso constante para determinar peso seco de la muestra (PS) (gr) y se estimó el porcentaje de humedad $(\% \mathrm{H})[\% \mathrm{H}=(\mathrm{PH}-$ $\mathrm{PS} / \mathrm{PH}$. Parte de las semillas recientemente colectadas (50 semillas), fueron evaluadas mediante el test de Tetrazolio (Moore, 1985), para determinar la viabilidad. Un lote de 100 semillas se dividió en cuatro muestras (25 semillas por muestra) y se colocaron en cajas herméticas con sílica gel en su interior, para favorecer la pérdida de humedad. Se efectuó un registro constante de la pérdida de humedad y se pusieron a germinar las muestras de semillas en la medida que alcanzaron 20, 15, 10 y $5 \%$ de contenido de agua, a la temperatura óptima de germinación hallada en el experimento anterior. Adicionalmente, a cada muestra de semilla, con diferente grado de humedad, se le efectuó la prueba de viabilidad con Tetrazolio. Las semillas estaban muy dañadas por insectos, lo que condicionó el tamaño de las muestras.

Tabernaemontana catharinensis: se evaluaron tres tratamientos de temperaturas constantes de incubación: 1) $20^{\circ} \mathrm{C}$, 2) 25 ${ }^{\circ} \mathrm{C}$ y 3) $30^{\circ} \mathrm{C}$. Se efectuaron recuentos periódicos durante 30 días, para establecer la fecha óptima que definió el porcentaje de germinación inicial y final. Para la evalua- ción del efecto de la temperatura en el comportamiento germinativo se procedió del mismo modo que en $P$. elegans.

\section{Análisis de datos}

Se realizó un Análisis de la Varianza $(\mathrm{p}<0,05)$, para cada especie, para evaluar el porcentaje final de germinación (PFG) y el índice de velocidad de emergencia [(IVE), Maguire (1962)] a las diferentes temperaturas de germinación evaluadas. Adicionalmente, para $C$. urucurana, se evaluó el efecto de los tratamientos pre-germinativos como otro factor, además de la interacción con las temperaturas de germinación. Previo a los análisis se corroboró la distribución normal de los errores (test de Shapiro-Wilks modificado) y homogeneidad de varianzas. Las diferencias de medias se evaluaron a través del test Tukey $(\mathrm{p}<0.05)$. Para todos los casos se utilizó el paquete estadístico InfoStat (Di Rienzo et al., 2018).

\section{RESULTADOS Y DISCUSIÓN}

En Croton urucurana, el test de viabilidad determinó un 68,8 \% de semillas viables al momento de la evaluación. La Figura 2 muestra el porcentaje de germinación acumulada, en términos de semillas viables, en las diferentes temperaturas de germinación para los diferentes tratamientos pre-germinativos.

Se registró un mejor comportamiento en las semillas incubadas en temperaturas alternantes $\left(20-30{ }^{\circ} \mathrm{C}\right)$, independientemente de los tratamientos pre-germinativos. El inicio de la germinación se presentó a los 7-8 días de la incubación. El número de semillas germinadas aumentó hasta el día 16-17 y luego se estabilizó hasta el final del periodo de la evaluación. A los 10 días de 
N. Ghío et al.

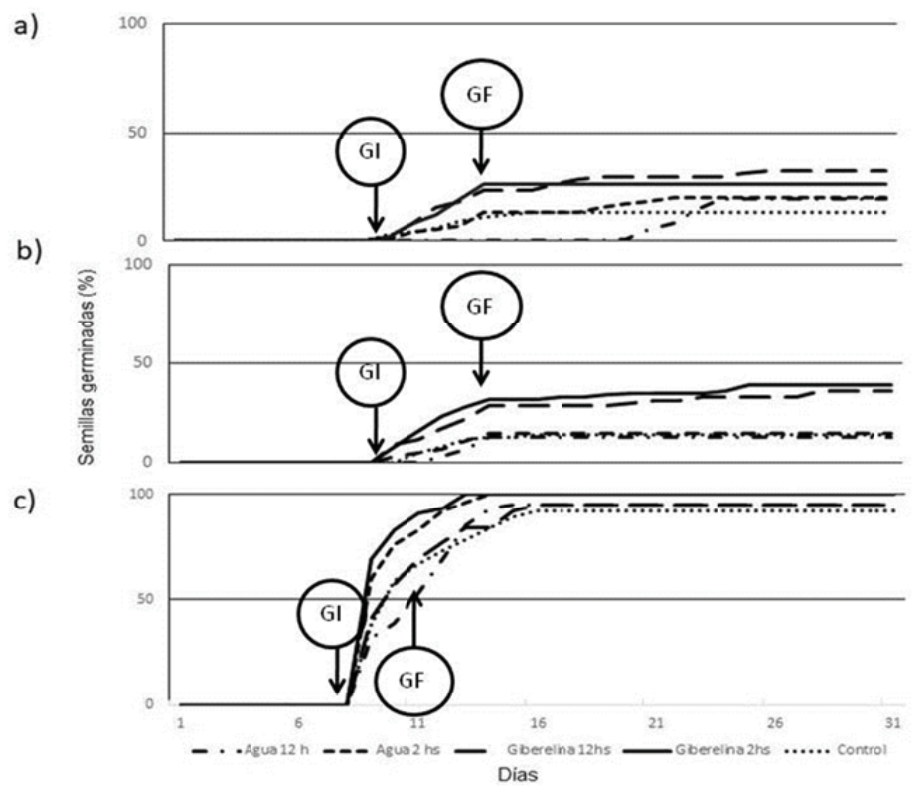

Figura 2: Velocidad de germinación. Germinación inicial (GI), Germinación final (GF) de C. urucurana a temperaturas constantes de a) $25^{\circ} \mathrm{C}$, b) $30^{\circ}$, c) alternancia $20-30^{\circ} \mathrm{C}$

Figure 2: Germination speed. Initial germination (GI), Final germination (GF) of C. urucurana at constant temperatures of a) $25^{\circ} \mathrm{C}$, b) $30^{\circ}$, c) alternation $20-30^{\circ} \mathrm{C}$

incubación se pudo observar el 50 \% de las semillas germinadas, mientras que a temperaturas de $25^{\circ} \mathrm{C}$ y $30^{\circ} \mathrm{C}$ no se alcanzó el 50 $\%$ de germinación ni al final del periodo de evaluación. A temperaturas sub-óptimas de germinación $\left(25\right.$ y $\left.30^{\circ} \mathrm{C}\right)$ se observó mayor velocidad de germinación en semillas de $C$. urucurana tratadas con giberelina (Figura 3).

En la Figura 3, se observa claramente la mejor respuesta hallada en condiciones de alternancia de temperaturas $\left(20-30{ }^{\circ} \mathrm{C}\right)$, independientemente de los tratamientos pre-germinativos evaluados. Por lo que se sugiere que, semillas de $C$. urucurana provenientes del centro-norte santafesino, no necesitan tratamientos pre-germinativos y la temperatura óptima de germinación se encuentra en temperaturas alternantes de
20-30 ${ }^{\circ} \mathrm{C}$, que es lo que se da normalmente en primavera en la región. A estas temperaturas, se sugiere realizar el recuento inicial de semillas germinadas a los 7-8 días de iniciada la incubación y el recuento final a los 16-17 días. Resultados similares han sido hallados por Scalon et al. (2012), en cuanto a tratamientos pregerminativos y temperatura óptima de germinación, para poblaciones de C. urucurana colectadas en Brasil.

En Paullinia elegans el test de viabilidad a través del ensayo bioquímico de Tetrazolio reveló el 95 \% de semillas viables para un porcentaje de humedad en semillas del $40 \%$ (inmediatamente luego de la recolección). La Figura 4 muestra la germinación acumulada de P. elegans a las diferentes temperaturas ensayadas, observándose mayor número 


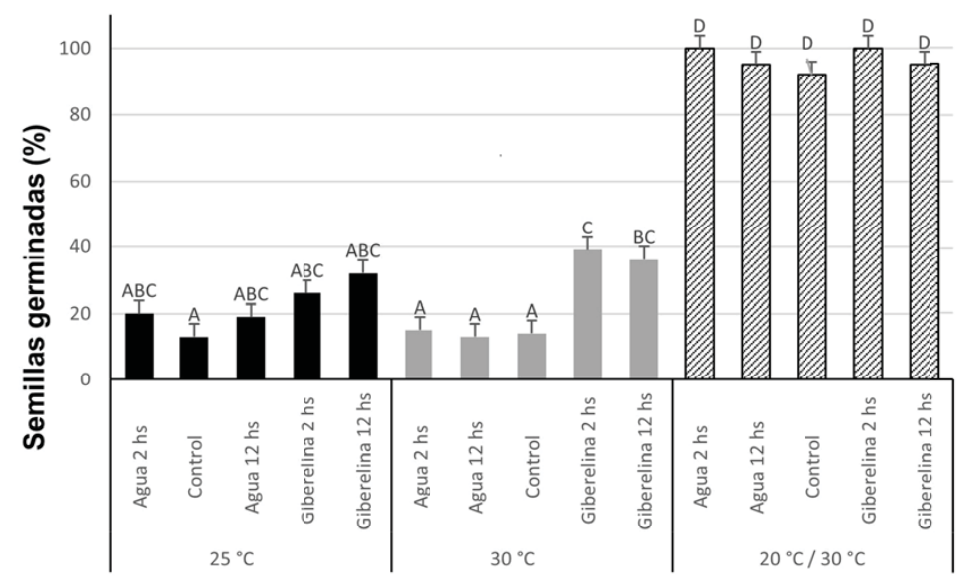

Tratamientos

Figura 3: Porcentaje de germinación acumulada de C. urucurana a temperaturas constantes de a) $25{ }^{\circ} \mathrm{C}$, b) $30{ }^{\circ} \mathrm{C}$ y c) alternancia $20-30{ }^{\circ} \mathrm{C}$.

Figure 3: Cumulative germination percentage of $C$. urucurana at constant temperatures of a) $25^{\circ}$ C, b) $30^{\circ} \mathrm{C}$ and c) alternation $20-30^{\circ} \mathrm{C}$.

Figura 4: Velocidad de germinación. Germinación inicial (GI), Germinación final (GF) de P. elegans a temperaturas constantes de a) $20^{\circ} \mathrm{C}$, b) $25^{\circ} \mathrm{C} \mathrm{y} \mathrm{C)}$ $30^{\circ} \mathrm{C}$.

Figure 4: Germination speed. Initial germination

(GI), Final germination (GF) of P. elegans at constant temperatures of a) $20^{\circ}$ C, b) $25^{\circ} \mathrm{C}$ and C) $30^{\circ} \mathrm{C}$. a)

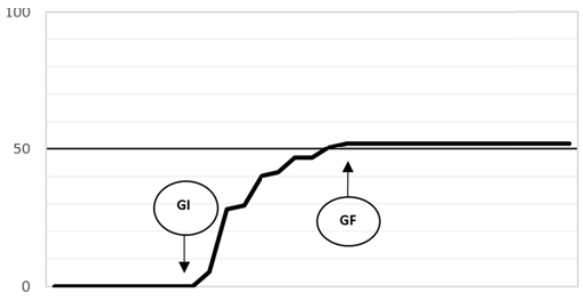

하)

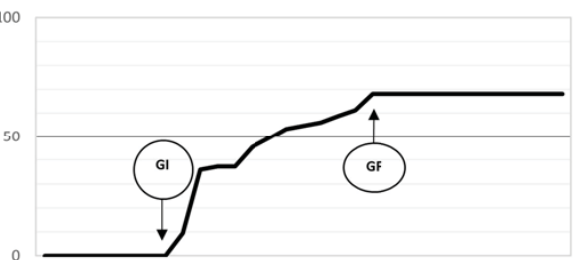

c)

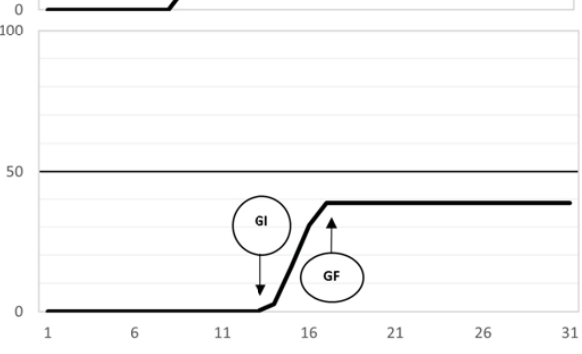

Días 
de semillas germinadas a $25^{\circ} \mathrm{C}$. El inicio de la germinación fue a 7 días de la incubación y a los 14 días germinaron el $50 \%$ de las semillas. El número de semillas germinadas continuó aumentando hasta los 19 días posteriores a la incubación, período en el cual se estabilizó hasta el final de la evaluación. En las semillas incubadas a $20^{\circ} \mathrm{C}$ si bien el día de inicio de germinación coincide con el de las semillas incubadas a $25^{\circ} \mathrm{C}$, éste se estabiliza a los 18 días con un porcentaje de germinación mucho menor. Con temperaturas de $30{ }^{\circ} \mathrm{C}$ de incubación no se alcanzó el 50 $\%$ de germinación de las semillas. Este efecto negativo del incremento de la temperatura en la velocidad de germinación puede tener relación con lo expuesto por Finch-Savage (2004), quien señala que, una vez llegado al nivel óptimo de temperaturas, donde la velocidad germinativa es mayor, ocurre un descenso a medida que las temperaturas se acercan a su límite máximo donde se produce un daño irreversible en las semillas.

Tanto para el PFG como para el IVE hallados en $P$. elegans, se observaron diferencias significativas entre las diferentes temperaturas de germinación $(p<0,0001$; $\mathrm{p}=0$,0176). El mayor PFG se registró en semillas incubadas a $25^{\circ} \mathrm{C}$, el cual ascendió al
$68 \%$. A una temperatura de incubación de $20{ }^{\circ} \mathrm{C}$ se registraron el $52 \%$ de las semillas germinadas, mientras que a $30{ }^{\circ} \mathrm{C}$ solo germinaron el $39 \%$ de las semillas. Del mismo modo el IVE fue mayor a $25^{\circ} \mathrm{C}(16,9)$, seguido del tratamiento a $20^{\circ} \mathrm{C}(12,9)$ y finalmente, el de $30{ }^{\circ} \mathrm{C}(7,0)$. El incremento de la temperatura tuvo un efecto negativo en la germinación, lo que coincide con lo señalado por Carvalho y Naka-gawa (2000), quienes indican que la germinación sólo ocurre apropiadamente dentro de un determinado rango de temperaturas. Se sugiere como temperatura optima de germinación para poblaciones de $P$. elegans nativas del centronorte santafesino, temperaturas constantes de $25{ }^{\circ} \mathrm{C}$, con una fecha de recuento inicial de semillas germinadas a 8-9 días de la incubación y una fecha final de 22-23 días.

En P. elegans, a través de la evaluación de viabilidad que se realizó a través del test de bioquímico de Tetrazolio, se evidencia que existe una relación directa entre la perdida de humedad de las semillas y su viabilidad; por lo que se sugiere $P$. elegans se trata de un tipo de semilla recalcitrante (Tabla 1). En especies afines como Paullinia cupana var. sorbilis (Mart.) Ducke, comúnmente conocida como Guaraná, en ensayos realizados

Tabla 1. Porcentaje de semillas viables de P. elegans. Test de Tetrazolio (1\%).

Table 1: Percentage of viable seeds of P. elegans. Tetrazolium test (1\%).

\begin{tabular}{|c|c|}
\hline $\begin{array}{c}\text { Contenido de humedad } \\
\text { en semillas (\%) }\end{array}$ & $\begin{array}{c}\text { Semillas } \\
\text { viables (\%) }\end{array}$ \\
\hline 40 & 95 \\
\hline 30 & 90 \\
\hline 20 & 30 \\
\hline 15 & 4 \\
\hline 10 & 0 \\
\hline 5 & 0 \\
\hline
\end{tabular}


por Carvalho (1982), los resultados obtenidos evidencian que las semillas de Guaraná se encuentran en el grupo de las llamadas semillas recalcitrantes, no soportando deshidratación severa, perdiendo su viabilidad extremadamente rápido cuando se almacena a granel o en paquetes porosos en condiciones ambientales naturales.

El conocimiento actual sobre la viabilidad y la capacidad de almacenamiento de semillas de $P$. elegans es limitado, nuestra experiencia nos permitió observar que la pérdida de humedad disminuye cuando se almacenaban los frutos en bolsas plásticas herméticas (tipo Ziploc ${ }^{\circledR}$ ) a bajas temperaturas $\left(4-8^{\circ} \mathrm{C}\right)$ en el transcurso de dos semanas Con respecto a guaraná, las obser- vaciones prácticas indican que las semillas comienzan a perder la viabilidad luego de 72 horas después de la cosecha cuando no se estratifican en aserrín húmedo (Calzavara, 1979).

En Tabernaemontana catharinensis se observó el 90,76 \% de semillas viables al inicio del ensayo. En cuanto al porcentaje de germinación acumulado, en la Figura 5 se observa que, semillas incubadas a $25^{\circ} \mathrm{C}$, comenzaron a germinar antes que a las restantes temperaturas (8 días). El número de semillas germinadas aumento hasta el día 17 y luego se estabilizó hasta el final del período de evaluación. A dicha temperatura a los 12 días de incubación se observó el $50 \%$ de las semillas germinadas; mientras
Figura 5: Porcentaje de germinación acumulada, Germinación inicial (GI) Germinación final (GF) de

T. catharinensis a temperaturas constantes de a) 20

${ }^{\circ} \mathrm{C}$, b) $25^{\circ} \mathrm{C} \mathrm{y} \mathrm{c)} 30^{\circ} \mathrm{C}$.

Figure 5: Percentage of accumulated germination,

Initial germination (GI)

Final germination (GF) of

T. catharinensis at constant temperatures of a) $20^{\circ} \mathrm{C}$, b) $25^{\circ} \mathrm{C}$ and c) $30^{\circ} \mathrm{C}$.

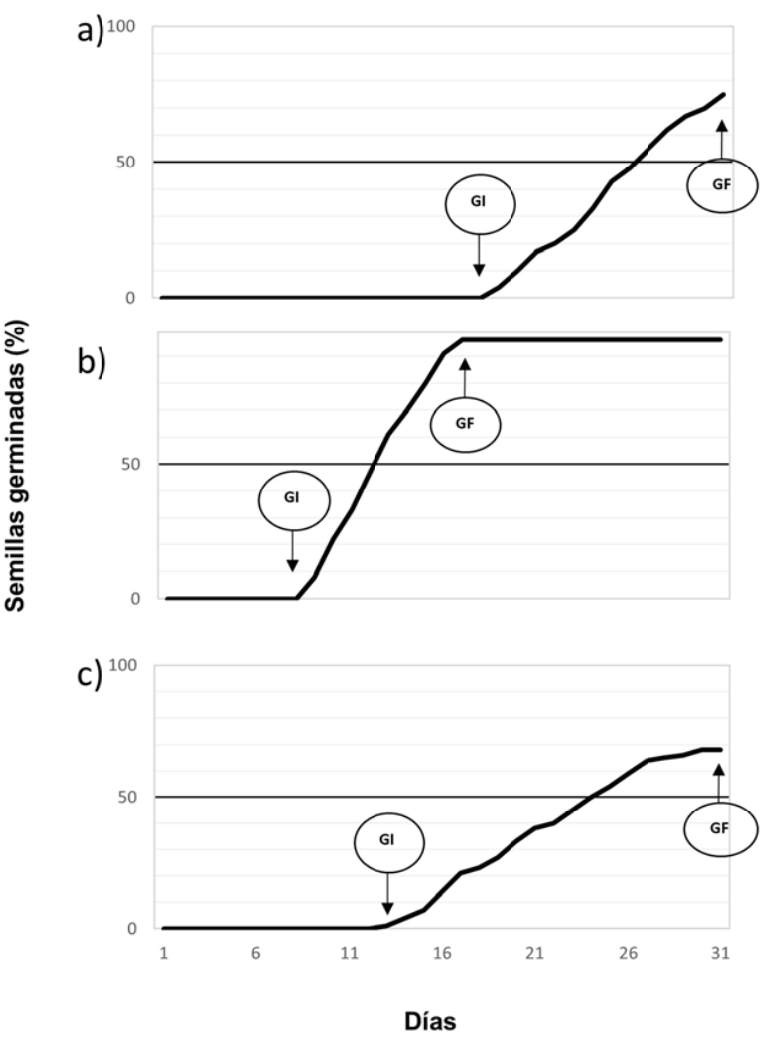


que se necesitaron de 26 días para alcanzar el $50 \%$ de semillas germinadas en incubación a $20^{\circ} \mathrm{C}$ y a $30{ }^{\circ} \mathrm{C}$ se necesitaron de 24 días de incubación. Afonso (2016) registró que la germinación de las semillas comenzó a los 15 días de incubación en poblaciones de Brasil.

Se hallaron diferencias significativas entre las diferentes temperaturas de germinación para el PFG y el IVE ( $\mathrm{p}<0,0001)$. Se registró el mayor porcentaje de germinación en semillas incubadas a $25^{\circ} \mathrm{C}$, presentando un $96 \%$ de poder germinativo. De la misma manera, la velocidad de germinación fue significativamente diferente en dicho tratamiento $(23,2)$, respecto a los restantes $\left(4,9\right.$ a $20^{\circ} \mathrm{C}$ y 7,7 a $30^{\circ} \mathrm{C}$ ) (Figura 5). Afonso (2016) registró resultados similares en poblaciones de T. catharinensis de la región noroeste de Rio Grande del Sur, Brasil. Por lo que se sugiere para T. catharinensis como temperatura óptima de germinación en poblaciones nativas del centro-norte de Santa Fe, temperaturas constantes de $25^{\circ} \mathrm{C}$ con una fecha de recuento inicial de semillas germinadas a 7-9 días de la incubación y una fecha final de 16-18 días.

\section{CONCLUSIONES}

El presente estudio permitió generar información valiosa sobre protocolos de germinación y conservación de semillas de tres especies nativas con potencial ornamental priorizadas para su introducción a cultivo en viveros: Croton urucurana, Paullinia elegans y Tabernaemontana cathariniensis. Esta información generará un impacto tecnológico inmediato relacionado con la producción de plantas ornamentales nativas en la zona de influencia de la Facultad de Ciencias Agrarias de la Universidad Nacional del Litoral y permitirá cubrir la actual demanda por especies nativas para uso paisajístico.

\section{AGRADECIMIENTOS}

Este trabajo se realizó dentro del Proyecto CAI+D Orientado 2016. Res. Consejo Superior No 632/17. Código: III - Sistema Productivo. 3.1. "INTRODUCCIÓN DE ESPECIES NATIVAS CON POTENCIAL ORNAMENTAL AL MERCADO FLORÍCOLA”.

\section{BIBLIOGRAFÍA}

Afonso MV. 2016. Seed technology and morphophysiological parameters in the propagation of Tabernaemontana catharinensis A. DC. (APOCYNACEAE). Dissertação (Mestrado em Ciências Biológicas) - Universidade Federal de Santa Maria, Santa Maria. [online] Disponible en: http://repositorio.ufsm. br/handle/1/4901. Acceso: 28/04/2018.

Biasatti NR, Rozzatti JC, Fandiño B, Pautaso A, Mosso E, Marteleur G, Vallejos L. 2016. Las ecoregiones, su conservación y las Áreas 
Naturales Protegidas de la provincia de Santa Fe. Ministerio de Medio Ambiente. Gobierno de Santa Fe. 244 pp. https://www. santafe.gov.ar/index.php/web/content/download/229660/1202209/file/LIBRO\%20ECOREGIONES_web.pdf

Biloni SJ. 1990. Árboles Autóctono Argentinos Tipográfica Editora Argentina. S.A. Pág 333.

Botto J, Mata D. 2014. Investigación científica y plantas ornamentales. Una mirada al futuro. Ciencia Hoy. Vol. 23. Nro 136. file://C:/ Users/Win7/Downloads/cienciahoyBotto_ Matavol136.pdf

Calzavara BBG. 1979. Orientación cultural del Guaraná. Belém, FCAP. (FCAP. Informe técnico $\left.n^{\circ} 2\right)$. Pág 53.

Carreras, ME, Ruiz GM, Bossa SR, Planchuelo AM. 2003. Desarrollo de una base de datos con especies de dicotiledóneas nativas de valor ornamental. XXIX Jornadas Argentinas de Botánica. Bol. Soc. Argent. Bot. 38 (Supl.), 253 p.

Carvalho, NM, Nakagawa, J. 2000. Sementes: ciência, tecnologia e produção. 4.ed. Jaboticabal: FUNEP. Pág. 588.

Di Rienzo JA, Casanoves F, Balzarini MG, Gonzalez L, Tablada MY, Robledo CW. 2018. InfoStat. Realese 2018. FCA - Universidad Nacional de Córdoba, Argentina.

Durigan G, Figliolia MB, Kawabata M, Garrido MA de O, Baitello JB. 1997. Sementes e mudas de árvores tropicais. São Paulo: Páginas Letras Editora e Gráfica. 65 p.

Finch-Savage WE. 2004. The use of population-based threshold models to describe and predict the effects of seedbed environment on germination and seedling emergence of crops. Pág 51-96. In R.L. Benech-Arnold, RL Sánchez (eds.). Seed physiology: applications to agriculture. Haworth Press, New York, USA.

Flora Argentina. 2019. Plantas vasculares de la República Argentina. [online] Disponible en: http://www.floraargentina.edu.ar/. Acceso: 01/11/2019.
Giménez AM, Moglia JG. 2003. Arboles del Chaco Argentino. Guía para el reconocimiento dendrológico. Pág. 310. Facultad de Ciencias Forestales, UNSE y Secretaría de Ambiente y Desarrollo Sustentable del Ministerio de Desarrollo Social. Editorial El Liberal.

Hartmann HT, Kester DE, Davies JRT, Geneve RL. 2002. Plant Propagation: Principles and practices. Seventh Edition. Prentice Hall. New Jersey. 880 p.

Knapp S. 2017. People and plants. The unbreakable bond. Humanos y Plantas. El vínculo indestructible. Conferencia Plenaria. Boletín de la Sociedad Argentina de Botánica. Volumen 52. Pp 86-87. Septiembre 2017. https://botanicaargentina.com.ar/wp-content/ uploads/2017/09/SAB-2018.pdf

Lorenzi H. 1992. Árvores Brasileiras: manual de identificação e cultivo de plantas arbó- reas nativas do Brasil. Nova Odessa, SP - Editora Plantarum.

Mori ES, Piña-Rodrigues FCM, Freitas NP, Martins RB. 2012. Sementes florestais: guia para germinação de 100 espécies nativas. São Paulo: Instituto Refloresta. 159 p.

Morisigue D, Mata D, Facciuto G, Bulrich L. 2012. Floricultura. Pasado y presente de la Floricultura Argentina. Instituto de Floricultura. Ediciones INTA. https://inta.gob.ar/ sites/default/files/script-tmp-inta_floricultura__pasado_y_presente_de_la_floricul.pdf

Moore RP. 1985. "Manual de ensayos al tetrazolio”. ISTA. Zurich. Edición española.

Pensiero JF, De la Peña M. 1999. Flora y avifauna de la Provincia de Santa Fe. Santa Fe. $384 \mathrm{p}$.

Pinho DS, Borges EEL, Corte VB, Nasser LCB. 2009. Avaliação da qualidade fisiológica de sementes de Anadenanthera peregrina (L.) Speg., durante o armazenamento. Rev Árvore 33: Pág 27-33. 
N. Ghío et al.

Rendón Correa A, Fernández Nava R. 2007. Scalon SP, Mussury RM, Lima AA. 2012. GerPlantas con potencial uso ornamental del Estado de Morelos, MÉXICO. 121-165, ISSN 1405-2768; México. http://www.scielo.org. mx/pdf/polib/n23/1405-2768-polib-23-121. pdf

REVINA. Red de Viveros de Plantas Nativas. http://reddeviverosdeplantasnativas.blogspot.com/2016/12/red-de-viveros-de-nativas-revina.html mination of Croton urucurana L. seeds exposed to different storage temperatures and pre-germinative treatments. Anais da Academia Brasileira de Ciências, 84(1), 191-200.

Verde Chaco. 2011. [online] Disponible: http:// arbolesdelchaco.blogspot.com.ar/2011/04/ ojo-de-muneca-falso-guarana.html. Acceso: 22/02/2016.

Willian RL. 1991. Capítulo 7. Almacenamiento de la semilla. Guía para la manipulación de semillas forestales (No. Fe 20/2). FAO. http://www.fao.org/3/ad232s/ad232s08.htm\#ch7. Acceso: 26/08/2020. 